PROCEEDINGS OF THE

AMERICAN MATHEMATICAL SOCIETY

Volume 139, Number 7, July 2011, Pages 2367-2373

S 0002-9939(2010)10679-2

Article electronically published on December 9, 2010

\title{
IRREDUCIBLE WEIGHT MODULES OVER WITT ALGEBRAS
}

\author{
XIANGQIAN GUO AND KAIMING ZHAO \\ (Communicated by Gail R. Letzter)
}

\begin{abstract}
In 1986, Shen defined a class of modules over the Witt algebra $W_{d}$ from irreducible modules over the general linear Lie algebra $\mathfrak{g l}_{d}$, which were also given by Larsson in 1992. In 1996. Eswara Rao determined the irreducibility of these modules. In this paper, we use simpler methods to give a short and straightforward proof to the results of Eswara Rao.
\end{abstract}

\section{INTRODUCTION}

Consider the Lie algebra $W_{d}$ of all derivations of the Laurent polynomial algebra $A=C\left[t_{1}^{ \pm 1}, t_{2}^{ \pm 1}, \ldots, t_{d}^{ \pm 1}\right]$. This algebra is known as the Witt algebra and is isomorphic to the algebra of diffeomorphisms of the $d$-dimensional torus. The algebra $W_{d}$ is a natural higher rank generalization of the Virasoro algebra; it has many applications to different branches of mathematics and physics (see [M2], [L1]- L5]) and at the same time a much more complicated representation theory.

Modules over Witt algebras were used by O. Mathieu [M2 to model simple cuspidal weight modules with finite-dimensional weight spaces over simple finitedimensional complex Lie algebras. Representations of Witt algebras are also closely connected to the representation theory of extended affine Lie algebras ( $\mathrm{AABGP}$ ) and toroidal Lie algebras ([B, E2, EJ]).

The representation theory of Witt algebras was intensively studied by many mathematicians and physicists; see [E1, E3, BMZ, L1, L2, L3, L4, L5, MZ, Z]. In particular, $\mathrm{MZ}$ asserted that any simple Harish-Chandra $W_{d}$-module is either dense (with uniformly bounded weight spaces) or punctured (with uniformly bounded weight spaces) or a simple quotient of some generalized Verma module. So far, the only known dense or punctured modules are those introduced and studied in ( $\underline{\mathrm{Sh}}, \mathrm{L} 3 \mathrm{]})$. The following conjecture is generally considered to be true.

Conjecture. All dense or punctured modules over $W_{n}$ are those mentioned in Theorem 1.

Assuming this conjecture and using the result in [BZ, we can deduce that the third class of modules mentioned above depends only on the first two classes of modules. So it is crucial to classify dense or punctured modules over $W_{n}$.

Eswara Rao [E1] determined the irreducibility of those modules introduced and studied in $(\underline{\mathrm{Sh}}, \underline{\mathrm{L} 3}])$. But we found that his proofs were very technical and hard

Received by the editors April 7, 2010 and, in revised form, June 23, 2010 and June 27, 2010.

2010 Mathematics Subject Classification. Primary 17B10, 17B20, 17B65, 17B66, 17B68.

Key words and phrases. Witt algebra, weight module, irreducible module.

(C) 2010 American Mathematical Society 
to understand. In this paper, we use much simpler and different methods to give a short and straightforward proof to the result in [E1].

We are grateful to the referee for giving a lot of suggestions to make the paper much more readable.

\section{Notation AND PRELIMINARIES}

2.1. Witt algebras $W_{d}$. We denote by $\mathbb{Z}, \mathbb{Z}_{+}$, and $\mathbb{C}$ the sets of all integers, nonnegative integers, and complex numbers, respectively.

We fix a positive integer $d>1$ and the column vector space $\mathbb{C}^{d}$ of $d \times 1$ matrices with the standard basis $\left\{e_{1}, e_{2}, \ldots, e_{d}\right\}$. Let $(\cdot \mid \cdot)$ be the standard symmetric bilinear form such that $(u \mid v)$ is the product $u^{T} v \in \mathbb{C}$, where $u^{T}$ is the matrix transpose.

Let $A=\mathbb{C}\left[t_{1}^{ \pm 1}, \ldots, t_{d}^{ \pm 1}\right]$ be the Laurent polynomial algebra over $\mathbb{C}$ and denote by $W_{d}$ the algebra of all derivations of $A$, called the Witt algebra. We set $t^{a}=$ $t_{1}^{a_{1}} t_{2}^{a_{2}} \cdots t_{d}^{a_{d}}$ for any $a \in \mathbb{Z}^{d}$ and $\partial_{i}=t_{i} \frac{\partial}{\partial t_{i}}$ for any $i \in\{1,2, \ldots, d\}$. For any $u \in \mathbb{C}^{d}$ and $r \in \mathbb{Z}^{d}$, we also set $D(u, r)=t^{r} \sum_{i=1}^{d} u_{i} \partial_{i}$ for convenience. The Lie bracket of $W_{d}$ is given by

$$
[D(u, r), D(v, s)]=D(w, r+s), \forall u, v \in \mathbb{C}^{d}, r, s \in \mathbb{Z}^{d},
$$

where $w=(u \mid s) v-(v \mid r) u$. Note that for any $u, v \in \mathbb{C}^{d}$, the product $u v^{T}$ is a $d \times d$ matrix.

Let $\mathfrak{t}=\left\{D(u, 0) \mid u \in \mathbb{C}^{d}\right\}$ be the Cartan subalgebra of $W_{d}$. A $W_{d}$-module $V$ is called a weight module if the action of $\mathfrak{t}$ on $V$ is diagonalizable. For any weight module $V$ we have the decomposition $V=\bigoplus_{\lambda \in \mathfrak{t}^{*}} V_{\lambda}$, where $\mathfrak{t}^{*}=\operatorname{Hom}_{\mathbb{C}}(\mathfrak{t}, \mathbb{C})$ and

$$
V_{\lambda}=\{v \in V: \partial v=\lambda(\partial) v \text { for all } \partial \in \mathfrak{t}\} .
$$

The space $V_{\lambda}$ is called the weight space corresponding to the weight $\lambda$. The support of the weight module $V$, denoted by $\operatorname{supp}(V)$, is defined as the set of all weights $\lambda$ with $V_{\lambda} \neq 0$. If $V$ is a weight $W_{d}$-module and $\operatorname{dim}_{\mathbb{C}} V_{\lambda}<\infty$ for all $\lambda \in \mathfrak{t}^{*}$, the module $V$ is called a Harish-Chandra module.

2.2. Finite-dimensional $\mathfrak{g l}_{d}$-modules. Let $\mathfrak{g l}_{d}$ be the Lie algebra of all $d \times d$ complex matrices, $\mathfrak{s l}_{d}$ the subalgebra of $\mathfrak{g l}_{d}$ consisting of all traceless matrices. For $1 \leq i, j \leq d$ we use $E_{i j}$ to denote the matrix units. Let $\mathfrak{H}=\operatorname{span}\left\{E_{i i} \mid 1 \leq i \leq d\right\}$ and $\mathfrak{h}=\operatorname{span}\left\{h_{i} \mid 1 \leq i \leq d-1\right\}$, where $h_{i}=E_{i i}-E_{i+1, i+1}$, and define $\epsilon_{i} \in \mathfrak{H}^{*}$ by $\epsilon_{i}\left(\sum_{j=0}^{d} a_{j} E_{j j}\right)=a_{i}$.

Let $P^{+}=\left\{\lambda \in \mathfrak{h}^{*} \mid \lambda\left(h_{i}\right) \in \mathbb{Z}_{+}, i=1,2, \ldots, d-1\right\}$. For any $\psi \in P^{+}$let $V(\psi)$ be the simple $\mathfrak{s l}_{d}$-module with highest weight $\psi$. We make $V(\psi)$ into a $\mathfrak{g l}_{d}$-module by defining the action of the identity matrix $I$ as some scalar $b \in \mathbb{C}$. We denote the resulting module as $V(\psi, b)$.

Define the fundamental weights $\delta_{i} \in \mathfrak{h}^{*}$ by $\delta_{i}\left(h_{j}\right)=\delta_{i, j}$ for all $i, j=1,2, \ldots, d-1$. It is well known that the module $V\left(\delta_{1}, 1\right)$ can be realized as the natural representation of $\mathfrak{g l}_{d}$ on $\mathbb{C}^{d}$ (the matrix product), which we can write as $E_{j i} e_{l}=\delta_{l i} e_{j}$. In particular,

$$
\left(r u^{T}\right) v=(u \mid v) r, \forall u, v, r \in \mathbb{C}^{d} .
$$

The exterior product $\bigwedge^{k}\left(\mathbb{C}^{d}\right)=\mathbb{C}^{d} \wedge \cdots \wedge \mathbb{C}^{d}$ (k times) is a $\mathfrak{g l}_{d}$-module with the action given by $X\left(v_{1} \wedge \cdots \wedge v_{k}\right)=\sum_{i=1}^{k} v_{1} \wedge \cdots v_{i-1} \wedge X v_{i} \cdots \wedge v_{k}$ for all $X \in \mathfrak{g l}_{d}$, 
and the following $\mathfrak{g l}_{d}$-module isomorphism is well known:

$$
\bigwedge^{k}\left(\mathbb{C}^{d}\right) \cong V\left(\delta_{k}, k\right), \forall 1 \leqslant k \leqslant d-1
$$

\section{Shen's mOdules $F^{\alpha}(\psi, b)$}

3.1. Defining the modules. Suppose $\psi \in P^{+}, b \in \mathbb{C}$ and $\alpha \in \mathbb{C}^{d}$. Denote $F^{\alpha}(\psi, b)=V(\psi, b) \otimes A$. For simplicity we write $v(n)=v \otimes t^{n}$ for any $v \in V(\psi, b)$ and $n \in \mathbb{Z}^{d}$. Then $F^{\alpha}(\psi, b)$ becomes a $W_{d}$-module by defining

$$
D(u, r) v(n)=(u \mid n+\alpha) v(n+r)+\left(r u^{T}\right) v(n+r),
$$

for all $u \in \mathbb{C}^{d}, n, r \in \mathbb{Z}^{d}, v \in V(\psi, b)$. The modules $F^{\alpha}(\psi, b)$ were defined in [Sh], L3 and studied in E1. We note that there are similarities shared by these modules and the generalized Verma modules studied in $\mathrm{KM}$.

One can easily verify that $F^{\alpha}(\psi, b) \simeq F^{\alpha+n}(\psi, b)$ for any $n \in \mathbb{Z}^{d}$. So we always assume that $\alpha_{i}=0$ if $\alpha_{i} \in \mathbb{Z}$ in what follows. Using (2.1) and (2.2) one can also verify that the modules $F^{\alpha}\left(\delta_{k}, k\right)$ for $k=1,2, \ldots, d-1$ have submodules

$$
W(\alpha, k)=\bigoplus_{n \in \mathbb{Z}^{d}}\left(\mathbb{C}(n+\alpha) \wedge \mathbb{C}^{d} \wedge \cdots \wedge \mathbb{C}^{d}\right) \otimes t^{n}
$$

If $\alpha=0$, then $F^{0}\left(\delta_{k}, k\right)$ for $k=1,2, \ldots, d-1$ have bigger submodules

$$
\tilde{W}(0, k)=W(0, k) \oplus\left(\mathbb{C}^{d} \wedge \cdots \wedge \mathbb{C}^{d}\right) \otimes t^{0} .
$$

For convenience, we also denote $\tilde{W}(\alpha, k)=W(\alpha, k)$ for $\alpha \neq 0$.

3.2. Eswara Rao's Theorem. We will give a much simpler proof for Eswara Rao's Theorem.

Theorem 1. Suppose $d>1, \psi \in P^{+} \backslash\{0\}, b \in \mathbb{C}$ and $k=1,2, \ldots, d-1$.

(a) If $(\psi, b) \neq\left(\delta_{k}, k\right)$, then $F^{\alpha}(\psi, b)$ is irreducible.

(b) $W(\alpha, k)$ and $F^{\alpha}\left(\delta_{k}, k\right) / \tilde{W}(\alpha, k)$ are irreducible, and

$$
F^{\alpha}\left(\delta_{k}, k\right) / \tilde{W}(\alpha, k) \simeq W(\alpha, k+1), \forall k=1,2, \ldots, d-1 .
$$

Proof. For convenience, we define $\mathbb{Z}_{*}^{d}(\alpha)=\left\{n \in \mathbb{Z}^{d}: n+\alpha \neq 0\right\}$ and $\mathbb{Z}_{* *}^{d}(\alpha)=$ $\left\{n \in \mathbb{Z}^{d}: n_{i}+\alpha_{i} \neq 0, \forall i=1, \ldots, d\right\}$. Let $M$ be a nonzero submodule of $F^{\alpha}(\psi, b)$ and denote $M_{n}=\left\{v \in V(\psi, b) \mid v \otimes t^{n} \in M\right\}$. Then $M_{n}$ is a subspace of $V(\psi, b)$ and $M=\bigoplus_{n \in \mathbb{Z}^{d}} M_{n} \otimes t^{n}$ since $M$ is a weight module. Denote $M_{*}=\bigcap_{n \in \mathbb{Z}_{*}^{d}(\alpha)} M_{n}$.

Claim 1. If $M_{*} \neq 0$, then $M=F^{\alpha}(\psi, b)$.

For any $v \in M_{*}, u \in \mathbb{C}^{d}, r \in \mathbb{Z}^{d}, n, n-k r \in \mathbb{Z}_{*}^{d}, k \in \mathbb{Z}$, from

$$
D(u, k r) v(n-k r)=\left((u \mid n-k r+\alpha) v+k\left(r u^{T}\right) v\right)(n)
$$

we see that $\left(r u^{T}\right) v \in M_{n}$; i.e., $\left(r u^{T}\right) v \in M_{*}$. So $M_{*}$ is a $\mathfrak{g l}_{d^{-}}$-submodule. Thus $M_{*}=V(\psi, b)$. If $\alpha \neq 0$, it follows that $M=F^{\alpha}(\psi, b)$.

Now suppose $\alpha=0$. For all $v \in V(\psi, b)$ and $i \neq j$ we have $D\left(e_{j},-e_{i}\right) v\left(e_{i}\right)=$ $-E_{i j} v(0)$, which implies that $\mathfrak{s l}_{d} V(\psi, b) \subset M_{0}$. Again it follows that $M=F^{\alpha}(\psi, b)$. Claim 1 is proved. 
We first prove (b). We identify $V\left(\delta_{k}, k\right)=\bigwedge^{k}\left(\mathbb{C}^{d}\right)$. For any $1 \leqslant k<d$ it is easy to see that the linear map

$$
\begin{gathered}
\pi_{k}: F^{\alpha}\left(\delta_{k}, k\right) \rightarrow F^{\alpha}\left(\delta_{k+1}, k+1\right), \\
\left(v_{1} \wedge v_{2} \wedge \cdots \wedge v_{k}\right)(n) \mapsto\left((n+\alpha) \wedge v_{1} \wedge v_{2} \wedge \cdots \wedge v_{k}\right)(n)
\end{gathered}
$$

is a $W_{d}$-module homomorphism. It is obvious that

$$
\begin{gathered}
\operatorname{ker}\left(\pi_{k}\right)=\tilde{W}(\alpha, k), \operatorname{Im}\left(\pi_{k}\right)=W(\alpha, k+1), \\
F^{\alpha}\left(\delta_{k}, k\right) / \tilde{W}(\alpha, k) \simeq W(\alpha, k+1)
\end{gathered}
$$

for all $k=1, \ldots, d-1$.

Recall that $W(\alpha, 1)=\bigoplus_{n \in \mathbb{Z}^{d}} \mathbb{C}\left((n+\alpha) \otimes t^{n}\right)$. Then by (3.1), we deduce that $D(u, r)\left((n+\alpha) \otimes t^{n}\right)=(u \mid n+\alpha)\left((n+r+\alpha) \otimes t^{n+r}\right)$, which implies the irreducibility of $W(\alpha, 1)$. Note also that $\tilde{W}(\alpha, d)=F^{\alpha}\left(\delta_{d}, d\right)$. Thus to obtain (b), we only need to show that $F^{\alpha}\left(\delta_{k}, k\right) / \tilde{W}(\alpha, k)$ are irreducible for $k=1, \ldots, d-1$.

Assume that $M$ properly contains $\tilde{W}(\alpha, k), 1 \leqslant k<d$. We may choose $\left(v_{1} \wedge v_{2} \wedge\right.$ $\left.\ldots \wedge v_{k}\right)(n) \in M_{n}(n) \backslash \tilde{W}(\alpha, k)$ for some $n \in \mathbb{Z}_{*}^{d}(\alpha)$, since $\tilde{W}(0, k)(0)=F^{0}\left(\delta_{k}, k\right)(0)$. It is clear that $n+\alpha, v_{1}, \ldots, v_{k}$ are linearly independent over $\mathbb{C}$. Take any $u \in \mathbb{C}^{d}$ such that $(u \mid n+\alpha) \neq 0$ and $\left(u \mid v_{i}\right)=0$ for all $i=1, \ldots, k$. Then

$$
D(u, r)\left(v_{1} \wedge v_{2} \wedge \ldots \wedge v_{k}\right)(n)=(u \mid n+\alpha)\left(v_{1} \wedge v_{2} \wedge \ldots \wedge v_{k}\right)(n+r),
$$

which implies that $v_{1} \wedge v_{2} \wedge \ldots \wedge v_{k} \in M_{n+r}$, i.e., $v_{1} \wedge v_{2} \wedge \ldots \wedge v_{k} \in M_{*}$. From Claim 1 we know that $M=F^{\alpha}(\psi, b)$. This proves (b).

Now we prove (a). Assume that $F^{\alpha}(\psi, b)$ is reducible. Then it suffices to show that $(\psi, b)=\left(\delta_{k}, k\right)$ for some $k=1, \ldots, d-1$. Recall that $M$ is a nonzero submodule of $F^{\alpha}(\psi, b)$.

To make the proof self-contained we include the following claim and its proof, which belong to Eswara Rao [E1, Lemma 3.2] with slight modifications.

Claim 2. If $v \in M_{n}$, then $M_{n}$ contains the following vectors: $\left(E_{i i}^{2}-E_{i i}\right) v, E_{i j}^{2} v$, $E_{i j} E_{i i} v,\left(n_{i}+\alpha_{i}\right) E_{j j} v-\left(n_{j}+\alpha_{j}\right) E_{j i} v$ for all $i \neq j$.

From (3.1) we see that

$$
\begin{gathered}
D(w,-r) D(u, r) v \otimes t^{n}=\left((w \mid n+\alpha+r)\left((u \mid n+\alpha) v+\left(r u^{T}\right) v\right)\right. \\
\left.-(u \mid n+\alpha)\left(r w^{T}\right) v-\left(r w^{T}\right)\left(r u^{T}\right) v\right) \otimes t^{n} \in M_{n}(n),
\end{gathered}
$$

for $u, w \in \mathbb{C}^{d}, r, n \in \mathbb{Z}^{d}, v \in M_{n}$.

Letting $w=u=r=e_{i}$ in (3.2), we obtain $\left(n_{i}+\alpha_{i}+1\right)\left(n_{i}+\alpha_{i}\right) v+E_{i i} v-E_{i i}^{2} v \in$ $M_{n}$, yielding $\left(E_{i i}-E_{i i}^{2}\right) v \in M_{n}$.

Letting $w=u=e_{j}$ and $r=e_{i}$ with $i \neq j$ in (3.2), we obtain that $\left(n_{j}+\alpha_{j}\right)^{2} v+$ $E_{i j}^{2} v \in M_{n}$, yielding $E_{i j}^{2} v \in M_{n}$.

Letting $w=u=e_{i}+e_{j}$ and $r=e_{i}$ with $i \neq j$ in (3.2), we simplify it to give $\left(\left(E_{i i}+E_{i j}\right)-\left(E_{i i}+E_{i j}\right)^{2}\right) v=\left(E_{i i}+E_{i j}-E_{i i}^{2}+E_{i j}^{2}-E_{i i} E_{i j}-E_{i j} E_{i i}\right) v \in M_{n}$.

Using the above-established results and the formula $E_{i j} v=\left(E_{i i} E_{i j}-E_{i j} E_{i i}\right) v$, we deduce that $E_{i j} E_{i i} v \in M_{n}$.

Letting $w=e_{i}$ and $u=r=e_{j}$ with $i \neq j$ in (3.2), and using the aboveestablished results, we deduce that $\left(n_{i}+\alpha_{i}\right) E_{j j} v-\left(n_{j}+\alpha_{j}\right) E_{j i} v \in M_{n}$. Thus, Claim 2 follows. 
Claim 3. If for some $n \in \mathbb{Z}_{* *}^{d}(\alpha), M_{n}$ contains a nonzero weight vector with respect to $\mathfrak{H}$, then $M=F^{\alpha}(\psi, b)$.

For any weight vector $v \in M_{n}$, from Claim 2 we know that $\left(n_{i}+\alpha_{i}\right) E_{j j} v-\left(n_{j}+\right.$ $\left.\alpha_{j}\right) E_{j i} v \in M_{n}$ for all $i \neq j$. But $\left(n_{i}+\alpha_{i}\right) E_{j j} v \in \mathbb{C} v$. Then $E_{j i} v \in M_{n}$ for all $i \neq j$. Thus $M_{n}$ is a nonzero $\mathfrak{s l}_{d}$-submodule, yielding that $M_{n}=V(\psi, b)$.

For any fixed $m \in \mathbb{Z}_{*}^{d}$, choose $u$ such that $(u \mid m+\alpha)(u \mid n+\alpha) \neq 0$. Then the algebra $\operatorname{Vir}(u, m-n)=\bigoplus_{i \in \mathbb{Z}} \mathbb{C} D(u, i(m-n))$ is isomorphic to the centerless Virasoro algebra and $\bigoplus_{i \in \mathbb{Z}} M_{n+i(m-n)}(n+i(m-n))$ is a uniformly bounded $\operatorname{Vir}(u, m-n)$ module. Notice that the weights of $M_{m}$ and $M_{n}$ relative to the algebra $\operatorname{Vir}(u, m-n)$ are $(u \mid m+\alpha) \neq 0$ and $(u \mid n+\alpha) \neq 0$ respectively. Then from the representation theory of the Virasoro algebra (see M1), we see that $\operatorname{dim} M_{m}=\operatorname{dim} M_{n}$. Thus $M_{m}=V(\psi, b)$ for all $m \in \mathbb{Z}_{*}^{d}$, and hence $M_{*}=V(\psi, b)$. Claim 3 follows from Claim 1.

For any $0 \neq v \in V(\psi, b)$ we can uniquely write $v=\sum_{\lambda \in \mathfrak{H}^{*}} v_{\lambda}$ such that $h v_{\lambda}=$ $\lambda(h) v, \forall h \in \mathfrak{H}$. Denote $\operatorname{Supp}(v)=\left\{\lambda: v_{\lambda} \neq 0\right\}$ and $\lambda_{i}=\lambda\left(E_{i i}\right), \forall \lambda \in \mathfrak{H}^{*}$. Define $S_{v}=\left\{i: \lambda_{i}=\mu_{i}, \forall \lambda, \mu \in \operatorname{Supp}(v)\right\}$. It is clear that $E_{i i} v \in \mathbb{C} v, \forall i \in S_{v}$.

Take any nonzero $v \in M_{n}, n \in \mathbb{Z}_{* *}^{d}(\alpha)$ such that $|\operatorname{Supp}(v)|$ is minimal. Denote $p=|\operatorname{Supp}(v)|$. Let

$$
K=\left\{u \in M_{n}: n \in \mathbb{Z}_{* *}^{d}(\alpha),|\operatorname{Supp}(u)|=p\right\} .
$$

From Claim 3 we know that $p \geqslant 2$ and $S_{u} \neq\{1,2, \ldots, d\}$ for any $u \in K$.

Claim 4. For any $v \in K$ and $\mu \in \operatorname{Supp}(v)$, the following holds:

(1) $\mu_{i} \in\{0,1\}$ for any $i$ and $1 \leqslant\left|\left\{i: \mu_{i}=1\right\}\right| \leqslant d-1$;

(2) $E_{i j} v_{\mu} \neq 0$ for some $i \neq j$ implies $i \in S_{v}$.

From Claim 2, we have $\left(E_{i i}^{2}-E_{i i}\right) v=\sum_{\mu \in \operatorname{Supp}(v)}\left(\mu_{i}^{2}-\mu_{i}\right) v_{\mu} \in M_{n}$. By the minimality of $|\operatorname{Supp}(v)|$, we must have that $\mu_{i}=\nu_{i}$ or $\mu_{i}+\nu_{i}=1, \forall \mu, \nu \in$ $\operatorname{Supp}(v), i=1,2, \ldots, d$.

For any $i \neq j$ with $i \notin S_{v}$, we claim that $E_{i j}^{2} v=E_{j i}^{2} v=0$. We have $E_{i j}^{2} v, E_{j i}^{2} v \in$ $M_{n}$ by Claim 2. If $E_{i j}^{2} v \neq 0$, we would have $\operatorname{Supp}\left(E_{i j}^{2} v\right)=\left\{\mu^{\prime}=\mu+2 \alpha_{i, j}: \mu \in\right.$ $\operatorname{Supp}(v)\}$ by the minimality of $\operatorname{Supp}(v)$. Since $i \notin S_{v}$ there are $\mu, \nu \in \operatorname{Supp}(v)$ with $\mu_{i} \neq \nu_{i}$ and hence $\mu_{i}^{\prime} \neq \nu_{i}^{\prime}$. On the other hand, $\mu_{i}^{\prime}+\nu_{i}^{\prime}=\mu_{i}+2+\nu_{i}+2=5 \neq 1$, contradicting the result in the last paragraph (with $E_{i j}^{2} v$ replacing $v$ ). So we have $E_{i j}^{2} v=0$. Similarly, we get $E_{j i}^{2} v=0$.

For any $i \neq j$ with $i \notin S_{v}$, we claim that $E_{i j} v_{\mu}=0$ if $\mu_{i} \neq 0$ and $E_{j i} v_{\mu}=0$ if $\mu_{j} \neq 0$, for any $\mu \in \operatorname{Supp}(v)$. We know that $E_{i j} E_{i i} v \in M_{n}$ from Claim 2. Similarly to the previous paragraph, one shows that $E_{i j} E_{i i} v=0$ and hence $E_{i j} E_{i i} v_{\mu}=0$, which implies that $E_{i j} v_{\mu}=0$ if $\mu_{i} \neq 0$. We can deduce that $E_{j i} v_{\mu}=0$ if $\mu_{j} \neq 0$ in the same way. Part (2) follows.

Since $p \geqslant 2$, for any $\lambda \in \operatorname{Supp}(v)$ with $\lambda_{i} \neq 0$ for some $i \notin S_{v}$ (such a $\lambda$ exists), we have the following arguments.

For any $i \neq j$ with $i \notin S_{v}$ and $\lambda_{i} \lambda_{j} \neq 0$, we have $E_{i j} v_{\lambda}=E_{j i} v_{\lambda}=0$ and hence $\left[E_{i j}, E_{j i}\right] v_{\lambda}=\left(E_{i i}-E_{j j}\right) v_{\lambda}=\left(\lambda_{i}-\lambda_{j}\right) v_{\lambda}=0$. Thus $\lambda_{i}=\lambda_{j}$. Since $p \geqslant 2$, in the case $\lambda_{1}=\lambda_{2}=\cdots=\lambda_{d} \neq 0$, there exists $\mu \in \operatorname{Supp}(v)$ with $\mu_{i}=0, i \notin S_{v}$ (otherwise $\mu=\lambda$ ). Thus $\lambda_{i}=1$ by $\mu_{i}+\lambda_{i}=1$ and hence $\lambda_{1}=\lambda_{2}=\cdots=\lambda_{d}=1$. Then $\mu_{1}+\cdots+\mu_{d}=d$ implies that $\mu_{j} \geq 2$ for some $j \neq i$, contradicting the fact that $\lambda_{j}=\mu_{j}$ or $\lambda_{j}+\mu_{j}=1$. So there exists some $\lambda_{j}=0$.

For $i \neq j$ with $i \notin S_{v}$ and $\lambda_{i} \neq 0, \lambda_{j}=0$, the facts that $E_{j, i}^{2} v_{\lambda}=0$ and $E_{i, j} v_{\lambda}=0$ imply that $0=\left[E_{i, j}, E_{j, i}^{2}\right] v_{\lambda}=2\left(\lambda_{i}-1\right) E_{j, i} v_{\lambda}=0$. Since $\left[E_{i, j}, E_{j, i}\right] v_{\lambda}=\lambda_{i} v_{\lambda} \neq 0$, 
then $E_{j, i} v_{\lambda} \neq 0$. We have $\lambda_{i}=1$. That is, $\lambda_{k} \in\{0,1\}$ for any $k=1, \ldots, d$. Since $\lambda_{k}=\mu_{k}$ or $\lambda_{k}+\mu_{k}=1$, we see that $\mu_{k} \in\{0,1\}$ for all $\mu \in \operatorname{Supp}(v)$. Claim 4 follows.

We define a total ordering $<$ on $\Lambda=\bigoplus_{i=1}^{d} \mathbb{Z} \epsilon_{i}$ as $k_{1} \epsilon_{1}+\cdots+k_{d} \epsilon_{d}<l_{1} \epsilon_{1}+\cdots+l_{d} \epsilon_{d}$ if there exists an $i$ such that $k_{j}=l_{j}$ for $j<i$ and $k_{i}<l_{i}$. Now fix any $v \in K$ such that $\lambda=\max (\operatorname{Supp}(v))$ is maximal with respect to this order.

We claim that $\lambda$ is the highest weight of $V(\psi, b)$, that is, $\psi=\lambda$. Otherwise, there is some $i$ such that $E_{i, i+1} v_{\lambda} \neq 0$. By Claim 4(2), we know that $i \in S_{v}$ and $E_{i i} v \in$ $\mathbb{C} v \subset M_{n}$. Then Claim 2 ensures that $\left(n_{i+1}+\alpha_{i+1}\right) E_{i i} v-\left(n_{i}+\alpha_{i}\right) E_{i, i+1} v \in M_{n}$ and hence $w=E_{i, i+1} v \in M_{n}$. By the minimality of $|\operatorname{Supp}(v)|$, we have $|\operatorname{Supp}(w)|=p$; that is, $w \in K$. However, it is clear that $\max (\operatorname{Supp}(w))=\lambda+\epsilon_{i}-\epsilon_{i+1}$, which is larger than $\max (\operatorname{Supp}(v))=\lambda$, contradicting the choice of $v$. So $\lambda=\psi$.

From Claim 4, we know that $\lambda_{i} \in\{0,1\}$. Since $\lambda$ is dominant, we must have $\psi=\lambda=\delta_{k}$, where $k$ is the number of $i$ with $\lambda_{i}=1$. Finally, $\left(\sum_{i=1}^{d} E_{i i}\right) v_{\lambda}=k v_{\lambda}$ implies $b=k$. This gives $(\psi, b)=\left(\delta_{k}, k\right)$ and completes the proof.

\section{ACKNowledgments}

The second author was partially supported by NSERC and NSF of China (Grant 10871192).

\section{REFERENCES}

[AABGP] B. N. Allison, S. Azam, S. Berman, Y. Gao, and A. Pianzola, Extended affine Lie algebras and their root systems, Mem. Amer. Math. Soc. 126 (605) (1997). MR.1376741 (97i:17015)

[B] Y. Billig, A category of modules for the full toroidal Lie algebra, Int. Math. Res. Not. 2006, Art. ID 68395, 46 pp. MR2272091(2007h:17030)

[BMZ] Y. Billig, A. Molev, and R. Zhang, Differential equations in vertex algebras and simple modules for the Lie algebra of vector fields on a torus, Adv. Math. 218(2008), no. 6, 1972-2004. MR2431666 (2009e:17042)

[BZ] Y. Billig and K. Zhao, Weight modules over exp-polynomial Lie algebras, J. Pure Appl. Algebra 191 (2004), no. 1-2, 23-42. MR.2048305 (2005a:17021)

[E1] S. Eswara Rao, Irreducible representations of the Lie-algebra of the diffeomorphisms of a $d$-dimensional torus, J. Algebra 182 (1996), no. 2, 401-421. MR1391590 (97f:17007)

[E2] S. Eswara Rao, Irreducbile representations for toroidal Lie-algebras, Journal of Pure and Applied Algebra 202 (2005), 102-117. MR2163403(2006e:17011)

[E3] S. Eswara Rao, Partial classification of modules for Lie algebra of diffeomorphisms of $d$ dimensional torus, J. Math. Phys. 45 (8) (2004), 3322-3333. MR2077512 (2005d:17028)

[EJ] S. Eswara Rao and C. Jiang, Classification of irreducible integrable representations for the full toroidal Lie algebras, J. Pure Appl. Algebra 200 (2005), no. 1-2, 71-85. MR 2142351 (2006b:17038)

[KR] V. Kac and A. Raina, Bombay lectures on highest weight representations of infinite dimensional Lie algebras, World Sci., Singapore, 1987. MR1021978 (90k:17013)

$[\mathrm{KM}] \quad$ O. Khomenko and V. Mazorchuk, Generalized Verma modules induced from $\mathrm{sl}(2, \mathbb{C})$ and associated Verma modules. J. Algebra 242 (2001), no. 2, 561-576. MR1848960 (2002f:17009)

[L1] T. A. Larsson, Multi-dimensional Virasoro algebra, Phys. Lett. B 231 (1989), 94-96. MR:1023577 (90k:17050)

[L2] T. A. Larsson, Central and non-central extensions of multi-graded Lie algebras, J. Phys. A 25 (1992), 1177-1184. MR 1154861 (93c:17047)

[L3] T. A. Larsson, Conformal fields: A class of representations of Vect $(N)$, Int. J. Mod. Phys. A 7 (1992), 6493-6508. MR1186508 (93h:17053)

[L4] T. A. Larsson, Lowest-energy representations of non-centrally extended diffeomorphism algebras, Commun. Math. Phys. 201 (1999), 461-470. MR.1682285 (2000c:17042) 
[L5] T. A. Larsson, Extended diffeomorphism algebras and trajectories in jet space, Commun. Math. Phys. 214 (2000), 469-491. MR1796031 (2001j:58017)

[M1] O. Mathieu, Classification of Harish-Chandra modules over the Virasoro Lie algebra, Invent. Math. 107 (1992), no. 2, 225-234. MR1144422 (93d:17034)

[M2] O. Mathieu, Classification of irreducible weight modules, Ann. Inst. Fourier (Grenoble) 50 (2000), no. 2, 537-592. MR:1775361 (2001h:17017)

[MZ] V. Mazorchuk and K. Zhao, Supports of weight modules over Witt algebras, Proc. R. Soc. Edinb. Sect. A-Math., in press.

[Sh] G. Shen, Graded modules of graded Lie algebras of Cartan type. I. Mixed products of modules, Sci. Sinica Ser. A 29 (1986), no. 6, 570-581. MR.862418 (88d:17014)

[Z] K. Zhao, Weight modules over generalized Witt algebras with 1-dimensional weight spaces, Forum Math. 16 (2004), no. 5, 725-748. MR2096685(2005h:17034)

Department of Mathematics, Zhengzhou University, Zhengzhou 450001, Henan, PeoPLE'S REPUBLIC OF CHINA

E-mail address: guoxq@amss.ac.cn

Department of Mathematics, Wilfrid Laurier University, Waterloo, On, Canada N2L 3C5 - and - College of Mathematics and Information Science, Hebei Normal University, Shijiazhuang, Hebei 050016, People's Republic of China

E-mail address: kzhao@wlu.ca 